2012 IEEE Ninth Electronics, Robotics and Automotive Mechanics Conference (CERMA). Rama estudiantil IEEE Universidad del sol, Cuernavaca, 2012.

\title{
Combining Color Constancy and Gamma Correction for Image Enhancement.
}

\section{Cepeda-Negrete, J. y Sanchez-Yanez, RE.}

Cita:

Cepeda-Negrete, J. y Sanchez-Yanez, RE. (2012). Combining Color Constancy and Gamma Correction for Image Enhancement. 2012 IEEE Ninth Electronics, Robotics and Automotive Mechanics Conference (CERMA). Rama estudiantil IEEE Universidad del sol, Cuernavaca.

Dirección estable: https://www.aacademica.org/jcepedanegrete/3 ARK: https://n2t.net/ark:/13683/pa8v/bow

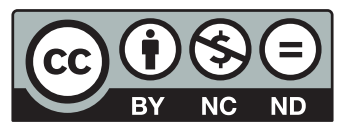




\title{
Combining Color Constancy and Gamma Correction for Image Enhancement
}

\author{
Jonathan Cepeda-Negrete and Raul E. Sanchez-Yanez \\ Universidad de Guanajuato DICIS \\ Carretera Salamanca-Valle de Santiago Km 3.5+1.8, Comunidad de Palo Blanco, Salamanca, Mexico \\ e-mail: jonathancn@laviria.org, sanchezy@ugto.mx
}

\begin{abstract}
In this paper, image enhancement issues are addressed by analyzing the effect of two well-known color constancy algorithms in combination with gamma correction. Those effects are studied applying the algorithms separately and in combination. Images from the Barnard dataset and from the Berkeley dataset are considered for experimental tests. The performance of the approaches is evaluated comparing the Average Power Spectrum Value of the test images and their corresponding outcomes, as a quality measure. According to the experimental results, it is observed that the application of the gamma correction after a color constancy algorithm results in an improved image quality.
\end{abstract}

Keywords-Gamma correction; color constancy; Retinex; gray world; power spectrum.

\section{INTRODUCTION}

Color is an important cue for computer vision and image processing related topics, like feature extraction [1], humancomputer interaction [2], and color appearance models [3]. Colors observed in images are determined by the intrinsic properties of objects and surfaces, as well as the color of the illuminant. For a robust color-based system, the effects of the illumination should be filtered out [4].

Color Constancy is the ability to recognize the correct colors, independently of the illuminant present in the scene [5]. Human vision has a natural ability to correct the color effects of the light source [3]. However, the mechanism that is involved in this capability is not yet fully understood [4]. The same process is not trivial to machine vision systems in an unconstrained scene [6].

Due to the wide range of computer vision applications that require color constancy, it is impossible to have a unique solution for all cases [6]. In recent decades, many researchers have addressed the problem of color constancy by proposing a number of algorithmic approaches. Gijsenij [4] classifies the algorithms into three types: 1) statistical methods, 2) gamut-based methods, and 3) learning-based methods. Moreover, Ebner [7] divides the algorithms into two groups, according to the illumination type: uniform and nonuniform. Despite this classification, in most of the color constancy corrections, the dynamic range in the image is expanded.

Color constancy and gamma correction methodologies, are commonly used in practical applications requiring a dynamic range correction. This is, similarly to some color constancy approaches, researches upon gamma correction have been addressed in dynamic range correction for image enhancement, like the dynamic contrast [8] and high dynamic range applications [9] issues. Because both approaches, color constancy and gamma correction, have been used for dynamic range correction, we consider interesting to test the effect caused by applying jointly the two techniques for image enhancement.

In the present work, the combined effect of two wellknown color constancy algorithms and gamma correction, are discussed. Both color constancy algorithms, are considered as statistical methods and oriented to uniform illumination scenes. Firstly, the White Patch Retinex (WPR) [10], [11], [12], taking the illuminant as the maximum intensity value in each color channel. Secondly, the Gray World (GW) [13], assuming the illuminant as the average value in each color channel. Each algorithm is well suited for particular cases, although, both solve many color constancy problems for uniform illumination.

We propose the application of color constancy algorithms in combination with gamma correction for the improvement of the quality of the image. The results are evaluated using the power spectrum metric [14] as a quality image measure. Images considered in the experimental test series, are taken from two different datasets [15], [16].

The rest of this paper is organized as follows. In Section 2, the color constancy algorithms, WPR and GW, are presented. Moreover, the gamma correction and the metric to evaluate the performance of the approaches are also discussed. Section 3 includes the experimental results in the test series, and the observations from the data obtained. Finally, the concluding remarks are given in Section 4.

\section{Methodology}

In this study, the combined effect of two basic algorithms in the field of color constancy, the WPR and GW, are tested in combination with the gamma correction. The outcomes from four particular approaches are compared, as depicted in Fig. 1.

Basically, the approaches are applied to each color constancy algorithm, separately, as follows.

1) Gamma correction,

2) Color constancy, 


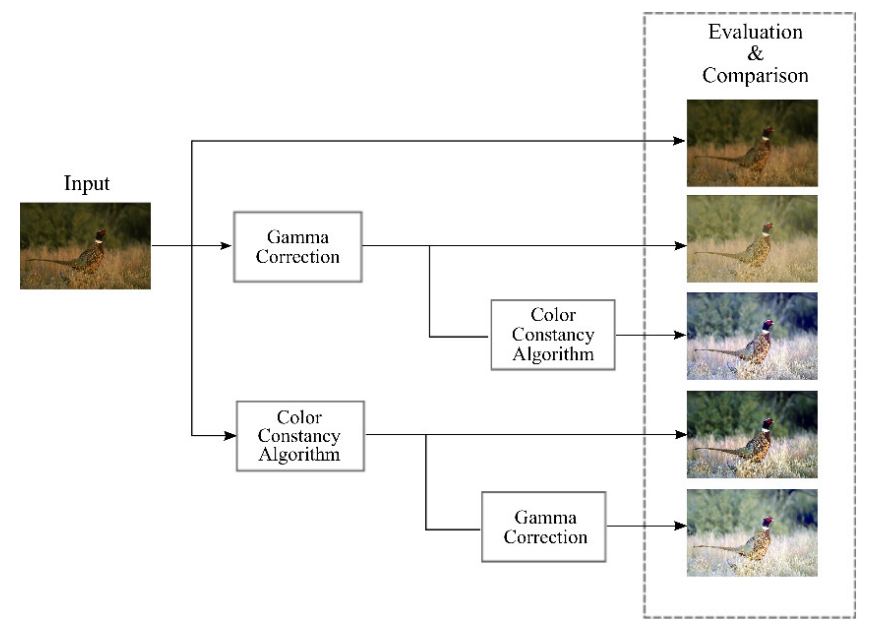

Figure 1. Experimental evaluation of four approaches combining color constancy and gamma correction algorithms.

3) Gamma correction and therefore, the color constancy algorithm,

4) Color constancy algorithm and afterwards the gamma correction.

After getting the resulting images, a quality measure for each input image and its corresponding outcomes is calculated. Finally, the five quality measures obtained are compared.

\section{A. Color constancy algorithms}

In this section, the WPR and GW color constancy algorithms are introduced. Furthermore, the basics relevant for the purposes of the work will be included.

The algorithms considered throughout this work assume that the illumination is uniform across the scene. Equation (1) gives the relationship for the color intensity,

$$
f_{i}(x, y)=G(x, y) R_{i}(x, y) I_{i},
$$

where, the $f_{i}(x, y)$ is the pixel intensity at position $(x, y)$ in an image or a frame, $G(x, y)$ is a factor that depends on the scene geometry, $R_{i}(x, y)$ is the reflectance of the object point displayed at position $(x, y)$ in the image and, $I_{i}$ is the illuminant in the scene. Index $i$ denotes the corresponding color channel in the image.

For color constancy algorithms with uniform illumination, it is assumed that $G(x, y)=1$ and $R_{i}(x, y)=1$, enabling the simplification

$$
f_{i}(x, y)=I_{i} .
$$

1) White Patch Retinex: The Retinex algorithm was proposed by Land and McCann [10]. In its simplest form, the White Patch Retinex, or WPR, takes into account the highest value in each color channel as the white representation for the image. Computationally, this white patch is found searching for the maximum intensity in each channel, and is given by

$$
I_{i_{\max }}=\max \left\{f_{i}(x, y)\right\} .
$$

Later, all pixel intensities are scaled using (4), according to the illuminant computed.

$$
o_{i}(x, y)=\frac{f_{i}(x, y)}{I_{i_{\max }}} .
$$

The WPR algorithm can be made more robust by computing a histogram $H_{i}$ for each color channel $i$. The histogram tells us how many image pixels have a particular intensity for a given color channel. Let $n_{b}$ be the number of bins of the histogram and $H_{i}(j)$ is the channel histogram, $j$ is the index of the corresponding intensity bin in the histogram. Instead of choosing the pixel with the maximum intensity for each color channel, one can choose the intensity such that all pixels with intensity higher than the one chosen account for some percentage of the total number of pixels. This method is used in [12] for shadow removal. Let $f_{i}(j)$ be the intensity of color channel $i$ represented by bin $j$ of the histogram $H_{i}$, then the estimate of the radiance is given by

$$
I_{i}=f_{i}\left(j_{i}\right),
$$

and, for the selection of the $j_{i}$, some conditions must be fulfilled:

$$
p n \leq \sum_{k=j_{i}}^{n_{b}} H_{i}(k) \quad \text { and } \quad p n \geq \sum_{k=j_{i}+1}^{n_{b}} H_{i}(k),
$$

where $p n$ is a percentage (usually about $1 \%$ ) of the total of pixels in the image [7].

2) Gray World: The gray world assumption is the best-known algorithm for color constancy. Proposed by Buchsbaum [13], it is used as reference for other algorithms. The GW is based on the assumption that, on average, the world is gray, and estimates the illuminant using the average color of all pixels. It is assumed that the information given by the average of each channel of the image is representative of the gray level.

The first step to do, is to compute the average color within the image, as is indicated in (7),

$$
a_{i}=\frac{1}{M N} \sum_{x=0}^{M-1} \sum_{y=0}^{N-1}\left\{f_{i}(x, y)\right\},
$$

where $M$ and $N$ are the number of columns and rows, respectively. Likewise, $a_{i}$ can be represented by

$$
\begin{aligned}
& a_{i}=\frac{1}{M N} \sum_{x=0}^{M-1} \sum_{y=0}^{N-1} G(x, y) R_{i}(x, y) I_{i}, \\
& a_{i}=I_{i} \frac{1}{M N} \sum_{x=0}^{M-1} \sum_{y=0}^{N-1} G(x, y) R_{i}(x, y),
\end{aligned}
$$




$$
a_{i} \approx I_{i} E\left[G R_{i}\right]=I_{i} E[G] E\left[R_{i}\right] .
$$

The function $E\left[G R_{i}\right]$ is the expected value of the geometry factor $G$ multiplied by the reflectance $R_{i}$. Both can be considered as independent random variables, as there is no correlation between the color and the shape of an object. Assuming that many different colors are present in the scene and each color is equally likely, the reflectance can be considered to be a random variable drawn from the range $[0,1]$, and

$$
\begin{gathered}
E[G] E\left[R_{i}\right]=E[G]\left(\int_{0}^{1} x d x\right)=E[G] \frac{1}{2}, \\
a_{i} \approx I_{i} E[G] \frac{1}{2} .
\end{gathered}
$$

Once that this global value is known, $I_{i}$, the illuminant, is computed. Assuming that there is a perpendicular orientation between the object and the camera, $E[G]=1$. Equation (13) describes the computation of the factor $\phi$ to be used later in (14).

$$
\begin{gathered}
\phi=\frac{2}{E[G]}=2 \\
I_{i} \approx \frac{2}{E[G]} a_{i}=\phi a_{i}
\end{gathered}
$$

Later, since $f_{i}(x, y)=G(x, y) R_{i}(x, y) I_{i}$, the output value is given by

$$
o_{i}(x, y)=\frac{f_{i}(x, y)}{I_{i}}=\frac{f_{i}(x, y)}{\phi a_{i}} .
$$

\section{B. Gamma Correction}

Most imaging systems produce values proportional to a radiance, that approximate red, green, and blue RGB tristimulus values. However, frequently these values are not proportional to intensity and are subject to a nonlinear transfer function. Gamma Correction (GC) can be thought of as the process of compensating the nonlinearity in order to achieve correct reproduction of relative luminance [17]. The luminance nonlinearity introduced by many imaging devices is described with an operation of the form

$$
g\left[f_{i}(x, y)\right]=f_{i}(x, y)^{\gamma},
$$

where $f_{i}(x, y) \in[0,1]$ denotes the image pixel intensity in the component $i$. If the value of $\gamma$ is known, then the inverse process is trivial

$$
g^{-1}\left[f_{i}(x, y)\right]=f_{i}(x, y)^{1 / \gamma} .
$$

The value of $\gamma$ is determined experimentally with the aid of a calibration target taking a full range of known luminance values through the imaging system [18]. However, often the calibration is not available or direct access to the imaging device is not feasible, as for example when downloading an image from the web. For many applications in digital photography, image processing, and computer vision, it would be advantageous correcting these nonlinearities, prior to the subsequent processing tasks. In practice, most imaging devices have a numerical value of gamma quite close to 2.5 [17].

Different transfer functions for $\mathrm{GC}$ have been standardized and are in use. Rec. 709, SMPTE 240M, sRGB and SDTV, are some transfer function examples. The notation sRGB [19] is widely used in images in personal computers, and for image exchange on the Internet. For this work, it is only used the sRGB transfer function for GC, given by

$$
g\left[f_{i}(x, y)\right]= \begin{cases}12.92 f_{i}(x, y) & f_{i}(x, y) \leq 0.0031308 \\ 1.055 f_{i}(x, y)^{\frac{1}{2 \cdot 4}} & f_{i}(x, y)>0.0031308\end{cases}
$$

\section{Image quality using Power Spectrum Metric}

In this study, the quality of an image is evaluated using the Power Spectrum Metric (PSM) [14]. The PSM determines the chromatic information in the image. Transforming an image into the frequency domain enables the analysis of characteristics of signals from another point of view. The discrete Fourier transform of the image $f(x, y)$ of size $M \times$ $N$ is defined by

$F(u, v)=\sum_{x=0}^{M-1} \sum_{y=0}^{N-1} \exp \left[-2 \pi i x \frac{u}{M}\right] \exp \left[-2 \pi i y \frac{v}{N}\right] f(x, y)$,

where $u, v$ are the frequency response components, being $x$ and $y$ the spatial components.

The Average Power Spectrum Value (APSV) is the mean value of the power spectrum in the image. This measure, used in this work as a quality index, is given by

$$
A P S V=\frac{1}{i} \sum_{i}|F|_{i}^{2},
$$

where $i$ stands for the color component in RGB and $|F|^{2}$ is given by

$$
|F|^{2}=\frac{1}{N M} \sum_{u=0}^{M-1} \sum_{v=0}^{N-1}|F(u, v)|^{2} .
$$

It is important to note that the APSV indicates the quality of each image. That is, the higher the APSV is, the better the quality is. Besides, the APSV is a no-reference image quality metric. In other words, it is not necessary to have any reference image for the evaluation. The APSV tends to be higher when the image shows a good illumination and numerous colors [20].

\section{EXPERIMENTAL RESULTS}

In this section, we present the experimental results obtained through this study. These results are presented separately for the GC and each color constancy algorithm. First, those for the WPR are given and afterwards those for GW. 
The image dataset selected for the experimental purposes consists of a significant number of 236 images. 70 images are taken from to the Barnard dataset [15], 148 images from the Berkeley dataset [16], and the remainders 18 are images taken from a particular collection or popular images from the web. It is important to note that, the 70 images belonging to the Barnard dataset are dark images under uniform illumination. The rest are natural images with nonuniform illumination.

Experimental results consist in a comparative analysis between the original image and outcomes from the four approaches for each color constancy algorithm, as indicated in Section 2. Therefore, $944(236 \times 4)$ resultant images are obtained. Thus, the APSVs were computed for the 944 outcomes and, additionally, for the 236 input images, getting a total of 1180 APSVs for the WPR study. 944 additional values are obtained for the $\mathrm{GW}$ tests series.

\section{A. Results for White Patch Retinex}

The image index for all images was previously arranged in ascendant order, according to the APSV (quality) of the input images. However, the APSVs for the other approaches have not an equal tendency, and their behavior is appreciably unsteady and varying. In order to clarify the tendency of results, the APSVs obtained were approximated by curve fitting. This approximation was computed using seven terms of Fourier Series, such that the curve fitted resembles the tendency of the particular approach, as shown in Fig. 2.

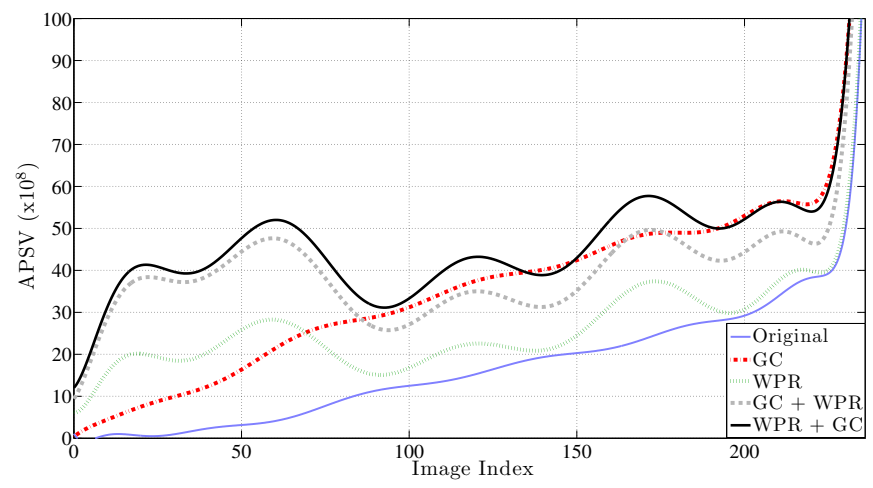

Figure 2. Tendency of the APSVs for each test image and its outcomes in the approaches using WPR.

In some cases, applying only the GC is better than adding the correction for the WPR. However, when the WPR and thereafter GC is applied, the results are generally, the best. According to the results, the difference is small between the combined approaches. Although, applying the WPR and afterwards, the GC is, without exception, the best.

Particularly, sample images of inputs and the outcomes are shown in Fig. 3 for the case of the WPR algorithm. First column contains the original images. Outcomes applying the algorithms are given in columns 2 to 5 , as indicated.

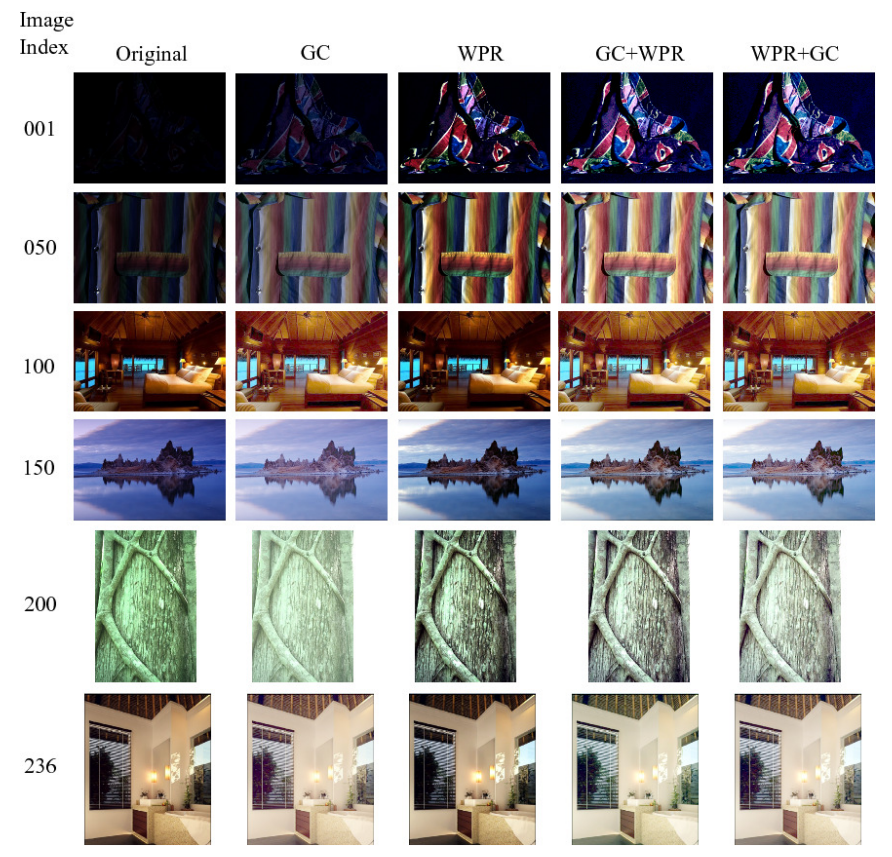

Figure 3. Six samples out of 236 images under test to WPR and their corresponding outcomes.

Table I

QUALITY MEASURES (APSV X10 ${ }^{8}$ ) FOR IMAGES IN FIG. 3.

\begin{tabular}{r||r|r|r|r|r}
\hline \hline $\begin{array}{r}\text { Img } \\
\text { idx }\end{array}$ & $\begin{array}{r}\text { Orig. } \\
\text { img }\end{array}$ & GC & WPR & $\begin{array}{r}\text { GC }+ \\
\text { WPR }\end{array}$ & $\begin{array}{c}\text { WPR }+ \\
\text { GC }\end{array}$ \\
\hline \hline 001 & 0.06 & 1.53 & 8.89 & 16.26 & $\mathbf{1 9 . 1 4}$ \\
\hline 050 & 2.95 & 22.18 & 26.97 & 55.93 & $\mathbf{6 1 . 0 6}$ \\
\hline 100 & 12.34 & 27.46 & 12.88 & 27.26 & $\mathbf{2 7 . 9 3}$ \\
\hline 150 & 20.48 & 43.33 & 26.69 & 40.53 & $\mathbf{4 8 . 4 5}$ \\
\hline 200 & 29.80 & $\mathbf{5 3 . 9 1}$ & 27.99 & 37.17 & 49.47 \\
\hline 236 & 64.14 & $\mathbf{1 1 3 . 2 0}$ & 63.59 & 105.82 & 111.99 \\
\hline \hline
\end{tabular}

Table I indicates the APSVs for each sample image. It is possible to appreciate that, consistently, the biggest numerical values are in the last column. Even in those cases when this value is not the biggest, it is close enough to the biggest one. This column includes the results for the outcomes applying the color constancy algorithm and thereafter GC.

\section{B. Results for Gray World}

Similarly as the WPR, the approaches were applied using the GW algorithm. Fig. 4 shows the results of the APSV in the dataset.

According to the results, for the first 70 images rearranged, the behavior using GW is similar to the WPR results. However, we can appreciate in Fig. 4 that, from the image 70 onwards, the APSVs on images maintain a similar value, particularly in the approaches using the GW algorithm. This behavior is observed, because these images contain a numerous amount of colors, and the GW algorithm 


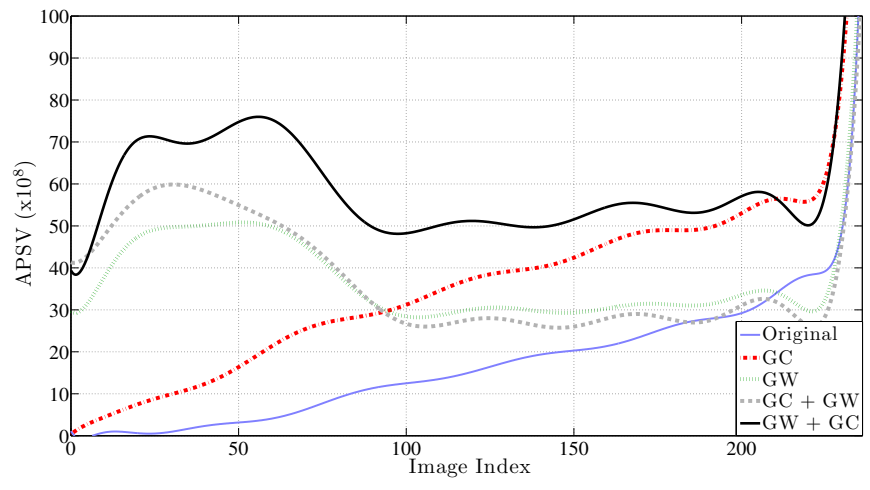

Figure 4. Tendency of the APSV for each test image and its outcomes in the approaches using GW.

calculates an average tending to gray level. In other words, for a set of grayish images the APSVs are quite similar. The results indicate that applying GW algorithm and thereafter $\mathrm{GC}$, is, as a rule, better than the other approaches.

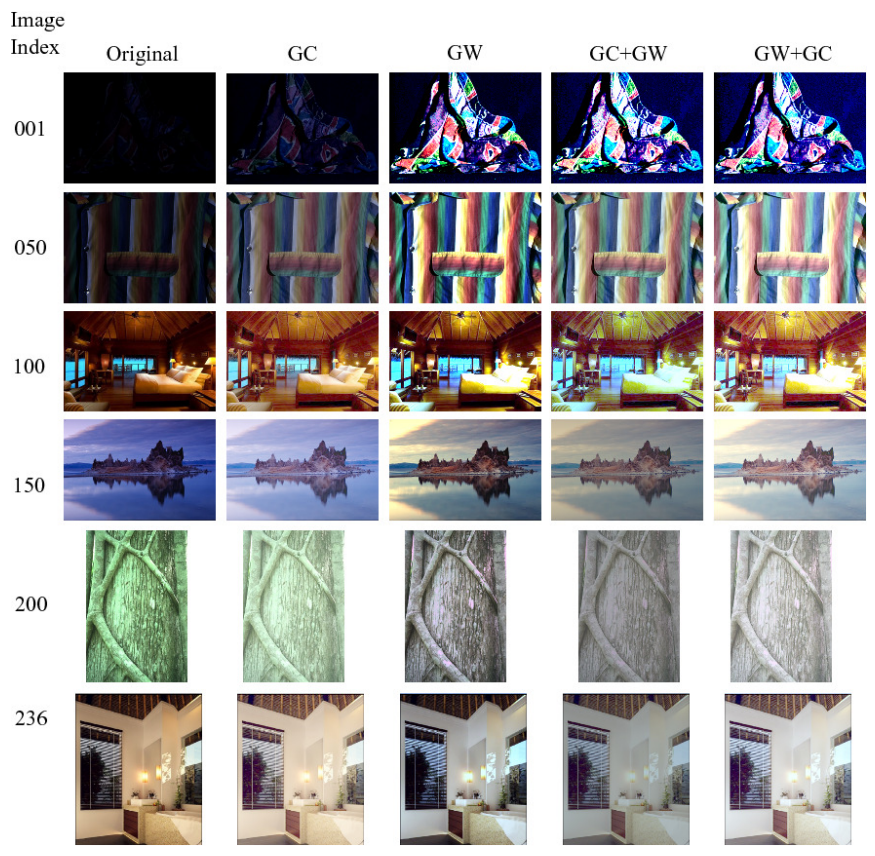

Figure 5. Six samples out of 236 images under test to GW and their corresponding outcomes.

Fig. 5 shows the same sample images previously indicated, but now, the approaches were applied using the GW algorithm. Furthermore, the Table II indicates the APSVs obtained for these images and its outcomes. We can appreciate that according to the results in the table, it is repeatedly better to apply the GW algorithm and afterwards GC. Similarly, to the WPR, in those cases when the APSV is not the biggest, the difference is narrow to the highest APSV.

Images belonging to the Barnard dataset, are contained in
Table II

QUALITY MEASURES (APSV X10 ${ }^{8}$ ) FOR IMAGES IN FIG. 5.

\begin{tabular}{r||r|r|r|r|r}
\hline \hline $\begin{array}{r}\text { Img } \\
\text { idx }\end{array}$ & $\begin{array}{r}\text { Orig. } \\
\text { img }\end{array}$ & GC & GW & $\begin{array}{r}\text { GC + } \\
\text { GW }\end{array}$ & $\begin{array}{l}\text { GW + } \\
\text { GC }\end{array}$ \\
\hline \hline 001 & 0.06 & 1.53 & 37.03 & $\mathbf{4 9 . 5 0}$ & 47.55 \\
\hline 050 & 2.95 & 22.18 & 58.35 & 57.11 & $\mathbf{9 4 . 0 8}$ \\
\hline 100 & 12.34 & 27.46 & 32.17 & 38.74 & $\mathbf{4 9 . 0 9}$ \\
\hline 150 & 20.48 & 43.33 & 29.30 & 26.33 & $\mathbf{5 2 . 5 7}$ \\
\hline 200 & 29.80 & $\mathbf{5 3 . 9 1}$ & 28.38 & 25.75 & 53.18 \\
\hline 236 & 64.14 & 113.20 & 91.00 & 80.53 & $\mathbf{1 3 6 . 4 0}$ \\
\hline \hline
\end{tabular}

the first 70 images rearranged. The behavior of the results in these images is different to the remainders because the former are dark images, and in this type of images the color correction increases the dynamic range. Thus, the APSVs are high due to the diverse chromatic information in the image. Furthermore, according to this study, the APSV corresponds to the human perception. In other words, the higher the APSV is, the better the quality perceived of the image is. We can appreciate in Figures 2 and 4 that, applying GW is generally better that WPR, especially, on dark images.

\section{CONClusions}

In the present work, the effect of two well-known color constancy algorithms in combination with gamma correction, were discussed for image enhancement purposes. The performance of the outcomes was measured by the average power spectrum value, as a quality image measure. A parallel goal of the application of the color constancy algorithms is the image enhancement, independently to the illuminant removed. According to our study, the approaches using WPR, as well as GW, increase the quality image to a great extent. It has been shown that gamma correction illuminates dark areas in the image, allowing a more clearly distinction of colors. Gamma correction is mainly used in practical applications requiring a dynamic range correction, an effect that also color constancy produces. Despite the image enhancement produced by a single algorithm, the combined application of a color constancy algorithm and afterwards the gamma correction, yields a better result. Finally, according to our study, it is suggested the use of gamma correction after a color constancy algorithm for dark image enhancement. Such improvement can be useful in a number of computer vision and image processing tasks.

\section{ACKNOWLEDGMENT}

Jonathan Cepeda-Negrete thanks to the Mexican National Council on Science and Technology, CONACyT, for the financial support provided via the scholarship 254884 (Grant No. 388681/290611).

\section{REFERENCES}

[1] T. Gevers and A. W.M. Smeulders, "Pictoseek: Combining color and shape invariant features for image retrieval," IEEE Trans. Image Process., vol. 9, no. 1, pp. 102-119, 2000. 
[2] J. Yang, R. Stiefelhagen, U. Meier, and A. Waibel, "Visual tracking for multimodal human computer interaction," in Proc. of the Conference on Human factors in computing systems, 1998, pp. 140-147.

[3] M. Fairchild, Color Appearance Models, H. Wiley, Ed. Wiley, Hoboken, 2005.

[4] A. Gijsenij, T. Gevers, and J. van de Weijer, "Computational color constancy: Survey and experiments," IEEE Trans. Image Process., vol. 20, no. 9, pp. 2475-2489, 2011.

[5] S. Zeki, A vision of the brain, J. Wiley and sons, Eds. WileyBlackwell, January 15, 1993.

[6] V. Agarwal, B. R. Abidi, A. Koshan, and M. A. Abidi, "An overview of color constancy algorithms," J. Pattern Recogn. Res., vol. 1, pp. 42-54, 2006.

[7] M. Ebner, Color Constancy, 1st ed., J. Wiley and Sons, Eds. Wrzburg, Germany: Wiley Publishing, 2007.

[8] J. Scott and M. Pusateri, "Towards real-time hardware gamma correction for dynamic contrast enhancement," in Applied Imagery Pattern Recognition Workshop (AIPRW), 2009 IEEE, 2009 , pp. $1-5$.

[9] Y. Cao and A. Bermak, "An analog gamma correction method for high dynamic range applications," in SOC Conference (SOCC), 2011 IEEE International, 2011, pp. 318 -322.

[10] E. H. Land and J. J. McCann, "Lightness and retinex theory," J. Opt. Soc. Am., vol. 61, no. 1, pp. 1-11, 1971.

[11] E. H. Land, "The retinex theory of color vision," Scientific American, vol. 237, no. 6, pp. 108-128, 1977.

[12] G. D. Finlayson, S. D. Hordley, C. Lu, and M. S. Drew, "On the removal shadows from images," IEEE Trans. Pattern Anal. Mach. Intell., vol. 28, no. 1, pp. 59-68, 2006.

[13] G. Buchsbaum, "A spatial processor model for object colour perception," Journal of The Franklin Institute, vol. 310, pp. $1-26,1980$.

[14] Y. Zhang, P. An, Q. Zhang, L. Shen, and Z. Zhang, "A noreference image quality evaluation based on power spectrum," in 3DTV Conference: The True Vision - Capture, Transmission and Display of $3 D$ Video (3DTV-CON), 2011, pp. 1-4.

[15] K. Barnard, L. Martin, B. Funt, and A. Coath, "A data set for color research," Color research and applications, vol. 27, no. 3, pp. 148-152, 2002.

[16] D. Martin, C. Fowlkes, D. Tal, and J. Malik, "A database of human segmented natural images and its application to evaluating segmentation algorithms and measuring ecological statistics," in Proc. 8th Int'l Conf. Computer Vision, vol. 2, July 2001, pp. 416-423.

[17] C. Poynton, Digital Video and HDTV Algorithms and Interfaces, 1st ed. San Francisco, CA, USA: Morgan Kaufmann Publishers Inc., 2003.
[18] C. Young-Chang and J. Reid, "RGB calibration for color image analysis in machine vision," IEEE Trans. Image Process., vol. 5, no. 10, pp. $1414-1422$, oct 1996.

[19] M. Stokes, M. Anderson, S. Chandrasekar, and R. Motta, "A standard default color space for the internet - sRGB," HewlettPackard, Microsoft, Tech. Rep., 1996.

[20] J. Cepeda-Negrete and R. E. Sanchez-Yanez, "Experiments on the white patch retinex in RGB and CIELAB color spaces," Acta Universitaria, vol. 22, no. NE-1, pp. 21-26, 2012. 